\title{
Evisceración omental transanal posterior a lesión de recto*
}

\author{
Drs. MARCO ALBÁN G. ${ }^{1,2}$, CARLOS JIMÉNEZ C. ${ }^{1}$, \\ ALEJANDRO ACUÑA Q. ${ }^{1}$, LUIS RUIZ B. ${ }^{1}$, CLAUDIA LEMUS R. ${ }^{1}$ \\ 1 Servicio de Urgencia Hospital San José. \\ 2 Departamento de Cirugía Hospital Clínico Universidad de Chile. \\ Santiago, Chile.
}

\begin{abstract}
\section{Omental evisceration through a rectal perforation. Case report}

Rectal perforation rarely occurs spontaneously. We report a 44 years old male consulting for abdominal pain lasting 12 hours. Abdominal muscular resistance was observed on physical examination. A plain abdominal X ray film showed a pneumoperitoneum. The patient was operated finding a perforation in the lower rectum, with omentum sliding through it and exiting through the anus. The omentum was sectioned and ligated and a derivative colostomy was performed. The cause of the rectal perforation was not ascertained.
\end{abstract}

Key words: Rectal perforation, omentum, evisceration.

\section{Resumen}

La perforación de recto se produce como resultado de una complicación bajo condiciones patológicas que comprometen la pared rectal. Rara vez ocurre espontáneamente. En casi todos los casos reportados de lesión rectal asociada a evisceración intestinal, el intestino delgado es el órgano involucrado. Las técnicas de reparación son variables, desde una rafia primaria en lesiones poco extensas, hasta resección de segmento lesionado y colostomía para los casos más graves. La decisión de la técnica a utilizar dependerá de la extensión y otras características de la lesión, del compromiso de estructuras vecinas, del tiempo de evolución y del grado de contaminación. El cirujano general debe estar preparado para el manejo de estas lesiones ya que son de resolución en los servicios de urgencias. Presentamos un caso, poco frecuente, de evisceración del epiplón mayor, transanal, posterior a lesión rectal de dudosa etiología.

Palabras clave: Lesión rectal, evisceración, epiplón mayor.

\section{Introducción}

La perforación de recto se produce como resultado de una complicación bajo condiciones pato- lógicas que comprometen la pared rectal, como la enfermedad diverticular, carcinoma, colitis, prolapso rectal crónico y causas traumáticas como contusión o trauma penetrante, lesiones intraluminales iatrogé-

*Recibido el 27 de julio de 2010 y aceptado para publicación el 11 de septiembre de 2010.

Correspondencia: Dr. Marco Albán G. Santos Dumont 999, Santiago, Chile. Fax: 56-2-9788335 marcoalban@gmail.com 


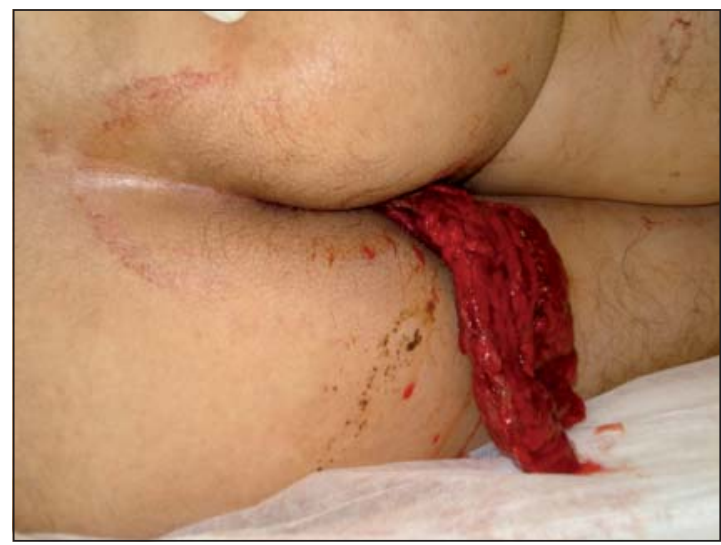

Figura 1. Evisceración transanal del epiplón mayor.

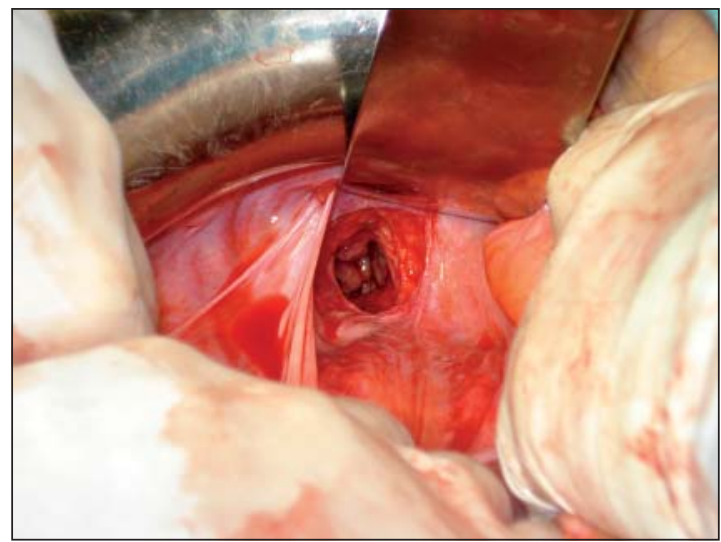

Figura 3. Lesión rectal.

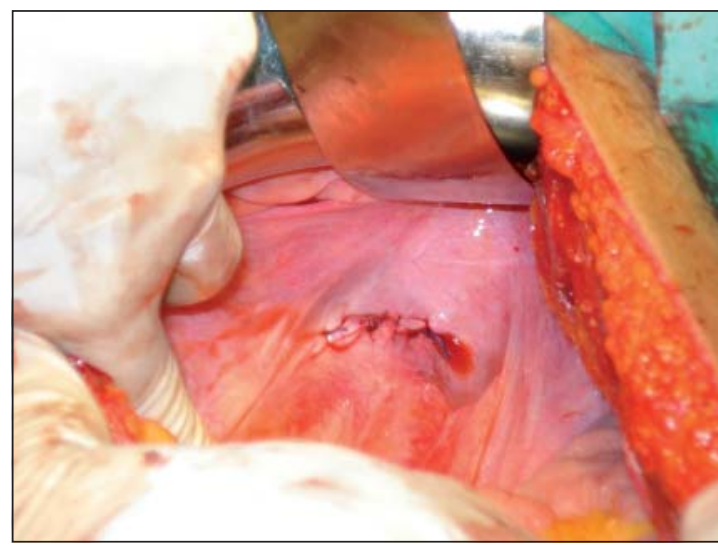

Figura 5. Lesión de recto suturada en dos planos con Vycril 3/0.

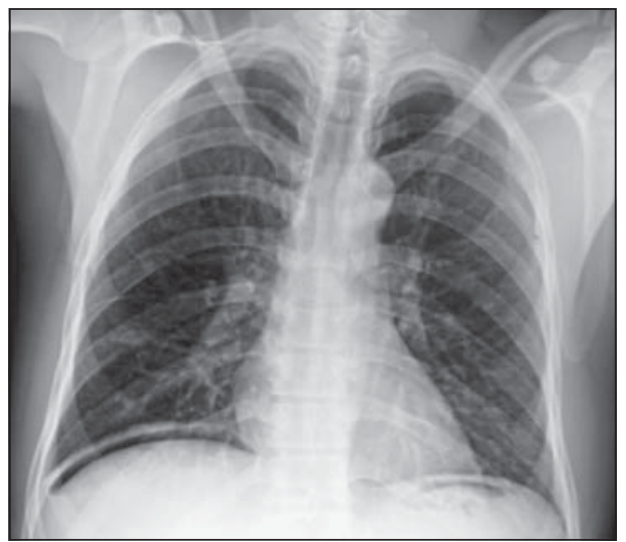

Figura 2. Neumoperitoneo en Rx.

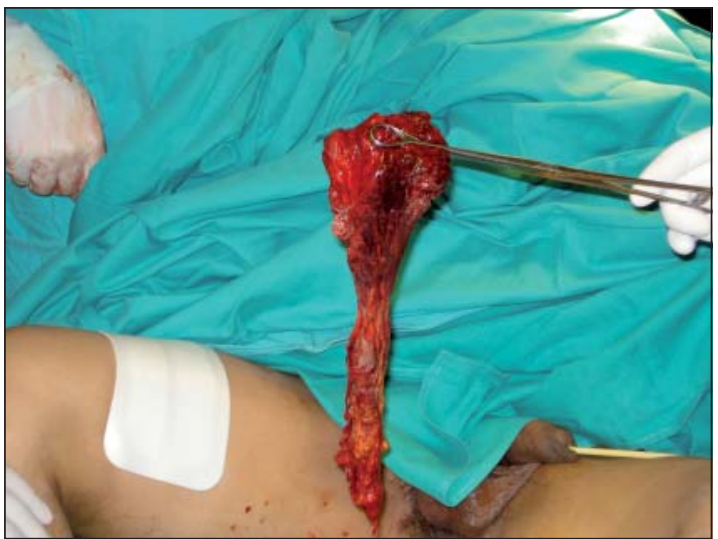

Figura 4. Retiro desde el exterior del segmento de epiplón mayor eviscerado, previo a su ligadura y sección intraabdominal antes de su trayecto a través de la lesión rectal.

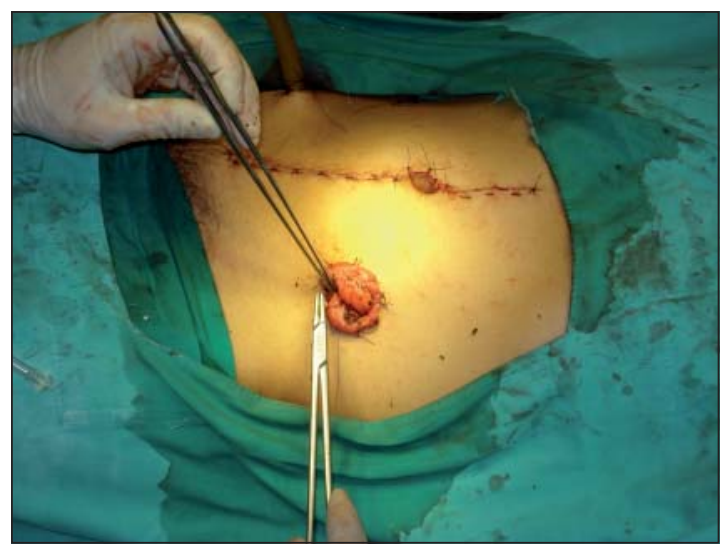

Figura 6. Colostomía en asa. 
nicas por instrumentación ${ }^{1}$ (reducción de prolapso, incisión en hemorroides), lesiones por cuerpo extraño². Rara vez ocurre espontáneamente ${ }^{3}$. Bajo las condiciones antes descritas, cualquier aumento de la presión intraabdominal o maniobra de Valsalva como defecación, vómitos, tos, contusión abdominal, se provoca una evisceración a través de la zona de la pared rectal comprometida ${ }^{4}$.

Casos más raros se describen en pediatría como el trauma por succión externa transanal por drenajes de piscinas o defectos congénitos de la pared rectal ${ }^{5}$.

En todos los casos reportados de lesión rectal asociada a evisceración, el intestino delgado es el órgano involucrado. En la literatura mundial existe sólo un caso reportado de evisceración omental ${ }^{6}$.

Nosotros presentamos un caso de evisceración transanal del epiplón mayor posterior a lesión rectal de dudosa etiología.

\section{Caso clínico}

Paciente masculino, 44 años, sin antecedentes mórbidos, consulta por cuadro de 12 horas de evolución, de dolor abdominal periumbilical, EVA 9/10, de inicio brusco, asociado a protrusión de masa adiposa transanal de características de epiplón mayor, posterior vómitos (Figura 1). Antecedente de caída por escalera el día anterior.

Al examen físico luce pálido, sudoroso, piel y mucosas deshidratadas, destacándose abdomen rígido, doloroso, resistencia muscular sin signos de irritación peritoneal. No presenta apremio ventilatorio ni descompensación hemodinámica. La radiografía de abdomen simple evidencia neumoperitoneo (Figura 2).

Ingresa con diagnóstico de abdomen agudo, epiplocele transanal.

Se realiza laparotomía exploradora a través de incisión en línea media supra e infraumbilical, se evidencia perforación anterior baja de recto de 2 a 3 $\mathrm{cm}$, con bordes vitales (Figura 3), a través de la cual se desliza epiplón mayor hacia recto y al exterior vía transanal. Las asas intestinales y resto del colon no presentaban lesiones ni estaban eviscerados, no se encontró cuerpo extraño, ni contaminación peritoneal.

La reparación quirúrgica se basó en ligadura y sección de epiplón en su segmento intraabdominal antes de su ingreso a recto a través de la lesión y retiro del mismo desde el exterior de su segmento eviscerado (Figura 4), rafia de lesión de pared anterior baja rectal con Vycril 3/0 en 2 planos (Figura 5), colostomía en asa derivativa (Figura 6) y aseo de cavidad peritoneal. Se dejó drenaje tubular en zona de sutura de lesión rectal.

\section{Discusión}

La lesión rectal o rectosigmoidea con evisceración del omento mayor es una situación excepcional de muy rara presentación, de hecho en la revisión realizada en MEDLINE y PUBMED sólo se encontró un caso publicado por Al-Abkari HA en el año $2000^{6}$. Las lesiones rectales asociadas a evisceración en casi todos los casos involucran a las asas intestinales.

Estas lesiones se pueden clasificar en 4 grupos de acuerdo con el sitio de la lesión y la presencia de daño del esfínter:

1. Perforación intraperitoneal sin lesión de esfínter.

2. Perforación intraperitoneal con lesión de esfínter.

3. Perforación extraperitoneal sin lesión de esfínter.

4. Perforación extraperitoneal con lesión de esfínter $^{7}$.

El mecanismo fisiopatológico tiene como base una lesión de la pared del recto por causa traumática, inflamatoria, isquémica, estructural o idiopática, que posterior a un aumento de la presión intrabdominal por tos, vómitos, defecación o contusión abdominal, provoca el deslizamiento a través de la lesión de estructuras intrabdominales que por lo general son asas intestinales, pero que en el caso presentado fue el epiplón mayor.

En adultos jóvenes el trauma directo de ano y recto se produce principalmente por traumatismos por proyectil, accidentes de tránsito, autoerotismo o abuso sexual ${ }^{8}$. El reporte de lesiones anorectales asociadas a prácticas sexuales han aumentado en frecuencia en la última década ${ }^{9}$.

En el caso reportado, la etiología no fue definida con exactitud, ya que los hallazgos en la cirugía no concordaban con la anamnesis, la lesión al parecer tenía pocas horas de evolución dada sus características de vitalidad en los bordes y sin presencia de contaminación peritoneal, además de no evidenciarse un compromiso isquémico del epiplón eviscerado ni lesión de otras estructuras intraabdominales. Lo cual no concuerda con la descripción del traumatismo previo al caer por las escaleras. Tampoco se encontraron fracturas ni lesiones en piel como equimosis, hematomas o aumento de volumen que apoyen más el mecanismo del trauma. El paciente no presentaba morbilidades asociadas y negó la posibilidad de lesión por cuerpo extraño, autoerotismo o prácticas sexuales que pudieran haber provocado el daño a nivel rectal.

Las lesiones traumáticas del recto representan un desafío para los cirujanos, debido a la singular anatomía de la zona. Desde su origen en el extremo distal del colon sigmoides hasta su terminación en el ano, el recto mide aproximadamente $15 \mathrm{~cm}$ de largo. 
Los dos tercios superiores del recto son intraperitoneales y el tercio inferior es extraperitoneal ${ }^{10}$.

La reparación primaria se recomienda para lesiones rectales superficiales aisladas, además son susceptibles de reparación transanal. Los pacientes con trauma penetrante abdominal con lesiones rectales complejas no susceptibles de reparación primaria deben ser tratados con colostomía de derivación. La colostomía en asa es segura y eficaz, la facilidad de realizarla y su cierre favorece su uso.

Si la lesión rectal es grave o lo suficientemente extensa como para requerir rectosigmoidectomía, se puede realizar una operación de Hartmann.

El uso de drenaje es innecesario en lesiones aisladas simples, pero puede ser útil en una lesión rectal extensa o grave o en pacientes con lesiones multisistémicas ${ }^{11}$. La decisión de la técnica a utilizarse para la reparación de la lesión rectal dependerá de la extensión y otras características de la lesión, del compromiso de estructuras vecinas, del tiempo de evolución y del grado de contaminación. El cirujano general debe estar preparado para el manejo de estas lesiones ya que son de resolución en los servicios de urgencias donde generalmente no existe coloproctólogo de turno.

\section{Referencias}

1. Goligher J, Duthie H, Nixon H. Spontaneous perforation of the normal large bowel. Surgery of the anus, rectum and colon. 5th edition. London: Bailliere Tindall, 1984;1133-4.

2. El-Ashaal YI, Al-Olama AK, Abu-Zidan FM. Trans-anal rectal injuries. Singapore Med J. 2008;49:54-6.

3. Shoab SS, Saravanan B, Neminathan S, Garsaa T. Thiersch Repair of a Spontaneous Rupture of Rectal Prolapse with Evisceration of Small Bowel through Anus - A Case Report. Ann R Coll Surg Engl. 2007;89:43-4.

4. Morris M, Setty S, Standage B, Hansen P. Acute Transanal Evisceration of the Small Bowel Report of a Case and Review of the Literature Dis Colon Rectum 2003;46:1280-3.

5. Cain WS, Howell CG, Ziegler MM, Finley AJ, Asch MJ, Grant JP. Rectosigmoid perforation and intestinal evisceration from transanal suction. J Pediatr Surg. 1983;18:10-3.

6. Al-Abkari HA. Spontaneous rupture of the rectum with evisceration of omentum through the anus: a case report and review of the literature. Ann Saudi Med. 2000;20:246-7.

7. Roche B, Michel JM, Deleaval J, Peter R, Marti MC. Traumatic lesions of the anorectum. Swiss Surg. 1998;5:249-52.

8. Joos AK, Herold A, Palma P, Post S. Perianal and rectal impalement injuries. Chirurg. 2006;77:781-9.

9. Orr CJ, Clark MA, Hawley DA, Pless JE, Tate LR, Fardal PMJ. Fatal anorectal injuries: a series of four cases. Forensic Sci. 1995;40:219-21.

10. McGrath V, Fabian TC, Croce MA. Rectal trauma: management based on anatomic distinctions. Am Surg. 1998;64:1136-41.

11. Herr M, Wascher R, Ronald A. Historical Perspective and Current Management of Traumatic Injury to the Extraperitoneal Rectum and Anus. Current Surgery 2005;62: 625-32. 